\title{
What Is the Most Useful Questionnaire for Measurement of Coping Strategies in Response to Nociception?
}

\author{
Joost T. P. Kortlever BSc, Stein J. Janssen MD, \\ Marijn M. G. van Berckel BSc, David Ring MD, PhD, \\ Ana Maria Vranceanu PhD
}

Published online: 24 June 2015

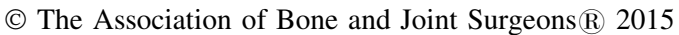

\begin{abstract}
Background There are several measures of coping strategies in response to nociception. These measures all correlate highly both with each other and with symptom intensity and magnitude of disability in patients with upper limb illness. This study aims to determine if distinct measures of coping strategies in response to nociception address the same underlying aspect of human illness behavior.

Questions/purposes Our primary study question was: is there one common aspect of human illness behavior measured by (1) the Pain Catastrophizing Scale (PCS); (2) the

Each author certifies that he or she, or a member of his or her immediate family, has no funding or commercial associations (eg, consultancies, stock ownership, equity interest, patent/licensing arrangements, etc) that might pose a conflict of interest in connection with the submitted article.

All ICMJE Conflict of Interest Forms for authors and Clinical Orthopaedics and Related Research ${ }^{\circledR}$ editors and board members are on file with the publication and can be viewed on request. Clinical Orthopaedics and Related Research ${ }^{\mathbb{R}}$ neither advocates nor endorses the use of any treatment, drug, or device. Readers are encouraged to always seek additional information, including FDAapproval status, of any drug or device prior to clinical use. Each author certifies that his or her institution approved the human protocol for this investigation and that all investigations were conducted in conformity with ethical principles of research.
\end{abstract}

J. T. P. Kortlever, S. J. Janssen, M. M. G. van Berckel, D. Ring Department of Orthopaedic Surgery, Orthopaedic Hand and Upper Extremity Service, Massachusetts General Hospital, Harvard Medical School, Boston, MA, USA

\section{A. M. Vranceanu $(\square)$}

Department of Psychiatry, Behavioral Medicine Service, Massachusetts General Hospital, Harvard Medical School, One Bowdoin Square, 7th Floor, Boston, MA 02114, USA e-mail: avranceanu@mgh.harvard.edu
Psychological Inflexibility in Pain Scale (PIPS); (3) the Patient-Reported Outcomes Measurement Information System-Pain Interference (PROMIS-PI) Computer Adaptive Test (CAT); and (4) the Pain Self-Efficacy Questionnaire (PSEQ)? Secondarily, we aimed to determine which of the four questionnaires is most psychometrically sound. We measured correlations among questionnaires, coverage, reliability, completion time, and collinearity of these questionnaires when entered together in a multivariable model with the shortened version of the Disabilities of the Arm, Shoulder and Hand (QuickDASH) upper extremity disability questionnaire.

Methods In this prospective study, 138 consecutive new or followup English-speaking patients aged 18 years or older presenting to a tertiary care referral center with traumatic and nontraumatic upper extremity conditions were invited to participate between March and May 2014. One hundred thirty-four (97\%) patients agreed to participate and completed the four questionnaires in random order before their visit with the physician. We used exploratory factor analysis to assess whether there was a single common trait-an underlying aspect of human illness behaviormeasured by these questionnaires. Interquestionnaire correlation was assessed using Spearman rank correlation coefficients; coverage by assessing floor and ceiling effect (proportion of scores at lower and upper limit); reliability by Cronbach's alpha measure of internal consistency; completion time in seconds using Kruskal-Wallis analysis; and collinearity statistics through a regression model with QuickDASH.

Results Exploratory factor analysis identified a common trait measured by these four measures-coping strategies in response to nociception-indicated by a substantial correlation of every individual questionnaire with the underlying trait (PCS: 0.74, PIPS: 0.84, PROMIS-PI: 0.83, PSEQ: 
-0.86). All interquestionnaire correlations were also large to substantial and were highest for PROMIS-PI with PSEQ (rho $=-0.84, p<0.001)$ and lowest for PROMIS-PI with PCS (rho $=0.67, \mathrm{p}<0.001$ ). Internal consistencies were high (PCS: 0.93, PIPS: 0.88, PSEQ: 0.92, and not determined for the PROMIS-PI as a result of its CAT administration). PROMIS-PI was the quickest to complete (30 seconds [interquartile range, 24-44]) compared with the others (PCS: 91 seconds [66-122], $\mathrm{p}<0.001$; PIPS: 105 seconds [82-141], $\mathrm{p}<0.001$; PSEQ: 78 seconds [60$101], \mathrm{p}<0.001)$. The four coping questionnaires had a low partial $\mathrm{r}^{2}$ and a relatively high variation inflation factor, indicating multicollinearity. PROMIS-PI was found to have the strongest correlation with QuickDASH ( $\beta$ coefficient: 0.63; standard error: 0.10; $p<0.001$ ).

Conclusions There is evidence that the four widely used measures of coping strategies in response to nociception address a single common aspect of human illness behavior, which negatively impacts upper extremity disability. Future studies assessing functional outcome should incorporate a measure of human illness behavior as it strongly relates to disability.

Clinical Relevance Given that all of these measures address the same important aspect of human illness behavior, we recommend the PROMIS-PI CAT as the most efficient measure.

\section{Introduction}

Pain is one of the most common presenting symptoms in the practice of a hand and upper extremity surgeon, highly influenced by coping strategies [16, 29]. There are several measures assessing ineffective pain coping strategies, often used in combination in research studies. Because collecting data can be a burden for both the researcher and the patient, finding the most effective, economical, and psychometrically sound measurement of coping with pain and its relationship to disability is important for both clinical and research practice.

The Patient-Reported Outcomes Measurement Information System (PROMIS)-Pain Interference computeradaptive test (CAT) is a novel measure of the degree to which pain limits an individual's physical, mental, and social activities [8], which indicates both difficulties with coping and disability. For hand and upper extremity conditions, pain intensity and magnitude of hand and armspecific disability are better explained by psychosocial factors such as stress, distress, and ineffective coping strategies rather than pathophysiology $[1,16,29]$. Ineffective coping strategies in response to pain are primarily measured using the Pain Catastrophizing Scale (PCS) [28], the Psychosocial Inflexibility in Pain Scale (PIPS) [31], and the Pain Self-Efficacy Questionnaire (PSEQ) [19]. Prior research has already noted a strong correlation between the PCS and the PSEQ [13]. Hence, using both measures of pain coping and measures of pain interference to assess coping and relationships with hand- and arm-specific disability may be redundant.

In our opinion, the questionnaires included in this study are used interchangeably to measure the impact of coping strategies in response to nociception on disability. Exploratory factor analysis is a statistical method that allows one to test such a hypothesis by bringing correlated variables together under a more general, underlying variable. In this way, redundancy among variables is reduced to an underlying trait (eg, construct or factor).

Exploratory factor analysis is a statistical method used to uncover the underlying-mathematical-structure of a set of variables. It is a technique used to describe variability among observed, correlated variables in terms of a potentially small number of unobserved variables called factors-in other words, to identify the underlying relationships between measured variables. This study addresses if these questionnaires measure a single common factor: coping strategies in response to nociception.

We evaluated whether exploratory factor analysis can identify a common underlying trait measured by (1) the PCS; (2) the PIPS; (3) the PROMIS-Pain Interference CAT; and (4) the PSEQ. Secondarily, we assessed the correlation among questionnaires to determine the amount of shared variance; compared instrument coverage and reliability; compared completion time; and compared collinearity of these questionnaires when entered together in a multivariable analysis of factors associated with the short version of the Disabilities of Hand Arm and Shoulder Questionnaire (QuickDASH).

\section{Patients and Methods}

After approval by the hospital's institutional review board, 138 new or followup patients presenting to a tertiary care referral center for hand and upper extremity conditions were invited to enroll in this cross-sectional study between March and May 2014. English-speaking patients aged 18 years or older presenting with traumatic and nontraumatic upper extremity conditions were invited to participate. Pregnant women were excluded as required by the institutional review board. Patients were enrolled before their visit with the physician. After obtaining informed consent, patients completed the questionnaires on a tablet, and data were collected through Assessment Center ${ }^{\mathrm{SM}}$ (Northwestern University Feinberg School of Medicine, Chicago, IL, USA, 2007). Assessment Center is a free, online data 
collection tool that enables researchers to create studyspecific websites for capturing participant data securely online. Customization of items or instruments (eg, formatting and randomization) is enabled by this web site [3]. Four patients (3\%) declined participation, leaving 134 patients for analysis. Patients were not compensated for participation.

\section{Sample Size Calculation}

Power analyses showed that a minimum sample size of 134 participants would provide 95\% statistical power (beta 0.05; alpha 0.05) to detect a correlation of 0.3 (moderate) on a bivariate correlation model between questionnaires.

\section{Outcome Measures}

Patient demographics and clinical factors were obtained first and consisted of: sex, age, ethnicity, race, education in years, work status, marital status, days since injury, prior visits for this condition, prior surgeries for this condition, other pain conditions (eg, migraine, low back pain, irritable bowel syndrome), and QuickDASH. Assessment Center made it possible to let the PCS, PIPS, PROMIS-Pain Interference, and PSEQ follow in a random order.

The QuickDASH is a shortened version of the in 1996 introduced 30-item Disabilities of Arm, Shoulder, and Hand (DASH) Outcome Measure questionnaire [10]. The QuickDASH uses 11 items, each answered on a 5-point Likert scale, to measure physical function and symptoms in persons with any or multiple musculoskeletal disorders of the upper limb. Scores range from 0 to 100 with higher scores indicating greater disability [4].

The PCS is a questionnaire used to quantify pain catastrophizing in patients with chronic pain initially developed in 1995 [28]. The PCS contains three dimensions. Domain 1, helplessness (questions 1-5 and 12), refers to the estimation that the person has not been able to do anything to influence the pain. Domain 2, magnification (questions 6, 7, and 13), refers to the exaggeration of the threatening properties of the painful stimulus. Domain 3, rumination (questions 8-11), refers to the fact that the patient cannot get rid of the idea of pain and cannot stop thinking about the pain. The PCS uses 13 items, each answered on a 5-point Likert scale. Scores range from 0 to 52 with higher scores indicating higher levels of catastrophizing [16, 18, 28].

The PIPS is one of the scales used for assessing relevant aspects of psychological inflexibility in pain patients, initially developed in 2008 [31]. The PIPS contains two dimensions. Domain 1, avoidance (questions 1, 2, 4, 5, 7, 8, 10 , and 11), measures the self-reported tendency to engage in certain behaviors that lead to avoidance. Domain 2, cognitive fusion related to pain (questions 3, 6, 9, and 12)cognitive fusion is a process whereby thoughts are taken at face value and believed as "facts" rather than "opinions"assesses the experienced frequency of acting on these presented thoughts as if they were true. The PIPS uses 12 items, each answered on a 7-point Likert scale. Scores range from 12 to 84 with higher scores indicating greater psychological inflexibility [25, 30, 31].

The PROMIS-Pain Interference is a computerized adaptive instrument that defines consequences of pain on relevant aspects of persons' lives and may include impact on social, cognitive, emotional, physical, and recreational activities as well as sleep and enjoyment of life. The questionnaire was initially developed in 2010 [2]. CAT provides a dynamic selection of items wherein the response to each item will guide the system's choice of the next item generating an individually tailored series of questions [2, 11, 12]. Each item reduces the potential for error and increases confidence in the score. The questionnaire will continue administering questions until the standard error drops below a specific level or when the patient has answered a maximum number of questions (set at 12 questions for this questionnaire). PROMIS-Pain Interference draws items from a 47-item bank, each answered on a 5-point Likert scale. The outcome scores are given in T-scores ranging from 39 to 84 with higher scores representing more of the concept being measured, greater pain interference. The T-score is set to have a mean of 50 and a SD of 10 . The advantage of this scoring approach is that a person's score communicates that person's level of the domain relative to the general population of the United States $[2,15]$ and allows comparison with other populations.

The PSEQ is a questionnaire that measures the influence of pain on confidence (self-efficacy) in performing tasks, initially developed in 1989. The PSEQ uses 10 items, each answered on a 7-point Likert scale. Scores range from 0 to 60 with higher scores indicating higher levels of self-efficacy [6, 19].

\section{Patient Demographics}

Patients were $57(43 \%)$ men and 77 women (57\%) with a median age of 52 years (interquartile range, 35-64 years) (Table 1). QuickDASH scores ranged from 0 to 91, reflecting inclusion of patients with no disability to severe symptoms and disability. The median time since onset of symptoms or injury before enrollment in the study was 90 days (interquartile range, 17-319 days).

\section{Statistical Analysis}

Exploratory factor analysis is a statistical method that looks for an underlying trait being measured by selected 
Table 1. Demographics $(n=134)$

\begin{tabular}{ll}
\hline Demographic & Median (IQR) \\
\hline Age (years) & $52(35-64)$ \\
Education (years) & $16(13-16)$ \\
Days since injury & $90(17-319)$ \\
QuickDASH & $31(14-50)$ \\
\hline
\end{tabular}

Number (\%)

Sex

Women

77 (57)

Men

Ethnicity

Non-Hispanic or Latino

Hispanic or Latino

$12(9.0)$

Race

White

Black or African American

Asian

Native Hawaiian or other Pacific Islander

Other or unknown

8 (6)

Work status

Working

Full-time

Part-time

$20(15)$

Other

Retired

$19(14)$

Unemployed, unable to work

Unemployed, able to work

$5(4)$

Homemaker

$4(3)$

Sick leave

2 (1)

Workers compensation

$1(1)$

Marital status

Married/living with partner

Single

Separated/divorced/widowed

Sought treatment before

$\begin{array}{ll}\text { No } & 83(62) \\ \text { Yes } & 51(38) \\ \text { Prior surgery } & \\ \text { No } & 117(87) \\ \text { Yes } & 17(13) \\ \text { Other pain conditions } & \\ \text { No } & 92(69) \\ \text { Yes } & 42(31)\end{array}$

$\mathrm{IQR}=$ interquartile range; QuickDASH $=$ short version of the Disabilities of the Arm, Shoulder and Hand (DASH) questionnaire.

variables and subsequently analyzes the correlation of these variables with the underlying trait. A factor score of 1 indicates a perfect association of the questionnaire with this underlying trait, 0 indicates no association, and -1 indicates perfect inverse association of the questionnaire with the underlying trait.

Interquestionnaire correlations to determine the amount of shared variance were measured using the Spearman rank correlation coefficient (range, -1 to 1 ); a score of 1 indicates a perfect correlation, 0 indicates no correlation, and -1 indicates a perfect inverse correlation.

Instrument coverage and reliability for each questionnaire were assessed by determining item completion rate, median total score with interquartile range, range, Cronbach alpha, and flooring and ceiling effect. Cronbach alpha is a measure of internal consistency (maximum score of 1) with a higher score indicating a higher internal consistency. Internal consistency using Cronbach alpha cannot be measured for PROMIS-Pain Interference because of its dynamic selection of items. This questionnaire works as a branching system wherein the response to each item will guide the system's choice of the next item generating an individually tailored series of questions for every patient. Flooring effect is a term used to describe a situation in which subjects in a study have scores at the lowest possible limit (floor); ceiling effect reflects scores at the upper limit (ceiling). Both effects demonstrate coverage of the questionnaire. Flooring and ceiling effect influences analysis because it reduces the amount of variation in a questionnaire.

A Kruskal-Wallis test was used to assess the difference in completion time among the four questionnaires. A Wilcoxon rank-sum (also known as Mann-Whitney) test was used to assess the difference in completion time between any two questionnaires. Bonferroni correction of the $\mathrm{p}$ value was used for the multiple comparisons in completion time.

A multivariable linear regression analysis with QuickDASH as the criterion was used to assess collinearity among the four pain-coping questionnaires. Multicollinearity was assessed with the partial $\mathrm{r}^{2}$, variance inflation factor (VIF), and tolerance (the reciprocal of VIF). The VIF is an index that measures how the variance of an estimated regression coefficient is increased because of collinearity. A low partial $r^{2}$ indicates that the questionnaire adds little to the already explained variance in outcome (QuickDASH) by the other explanatory variables.

Baseline characteristics are presented as median with interquartile range for continuous variables and as frequencies with percentages for categorical variables.

STATA 12.0 (StataCorp LP, College Station, TX, USA) was used for statistical analysis. A $p$ value $<0.05$ was considered to indicate statistical significance.

\section{Results}

We found that there was one underlying construct measured by all four questionnaires, namely, coping strategies 
in response to nociception. Exploratory factor analysis showed that three questionnaires (PCS: 0.74, PIPS: 0.84, and PROMIS-Pain Interference: 0.83) measure the same construct and one questionnaire measures the inverse of this construct (PSEQ: -0.86). This is indicated by a substantial correlation (ie, factor scores above 0.7 or below -0.7 ) of every individual questionnaire with the underlying trait (Table 2).

All interquestionnaire correlations were large to substantial; the lowest interquestionnaire correlation was seen between the PCS and PROMIS-Pain Interference (rho = $0.67, \mathrm{p}<0.001$, a large correlation) and the highest interquestionnaire correlation was that between the PROMIS-Pain Interference and PSEQ (rho $=-0.84, \mathrm{p}<0.001$, substantial correlation) (Table 3 ), suggesting a large

Table 2. Factor loadings from exploratory factor analysis measuring the underlying trait

\begin{tabular}{lc}
\hline Variable & Factor 1 (underlying trait) \\
\hline PCS & 0.7401 \\
PIPS & 0.8374 \\
PROMIS-PI & 0.8337 \\
PSEQ & -0.8587
\end{tabular}

PCS = Pain Catastrophizing Scale; PIPS = Psychological Inflexibility in Pain Scale; PROMIS-PI = Patient-Reported Outcomes Measurement Information System-Pain Interference; PSEQ = Pain SelfEfficacy Questionnaire.

Table 3. Spearman rank correlations among questionnaires

\begin{tabular}{lrrrr}
\hline Questionnaire & PCS & \multicolumn{1}{l}{ PIPS } & PROMIS-PI & PSEQ \\
\hline PCS & 1.0000 & & & \\
PIPS & 0.7037 & 1.0000 & & \\
PROMIS-PI & 0.6674 & 0.7093 & 1.0000 & \\
PSEQ & -0.7015 & -0.7028 & -0.8379 & 1.0000 \\
\hline
\end{tabular}

PCS = Pain Catastrophizing Scale; PIPS = Psychological Inflexibility in Pain Scale; PROMIS-PI = Patient-Reported Outcomes Measurement Information System-Pain Interference; PSEQ = Pain SelfEfficacy Questionnaire. $P$ values were below 0.001 for all correlations. amount of shared variance among these measures (redundancy).

Floor and ceiling effects (percentage of subject scores at the lower and upper limit) were seen in the PCS (21\% and $0.75 \%$, respectively); a floor effect was seen in the PIPS $(4.5 \%)$ and PROMIS-Pain Interference (12\%); and a ceiling effect was seen in the PSEQ (20\%) (Table 4).

There was high internal consistency in the PCS (0.93), PIPS (0.88), and PSEQ (0.92) indicating reliability of these questionnaires. Internal consistency is not an appropriate measure for the PROMIS-Pain Interference as a result of its CAT method.

The PROMIS-Pain Interference questionnaire takes the least amount of time to complete (30 seconds; interquartile range, 24-44), and the others required greater times (PCS: 92 seconds [interquartile range, 66-122], $\mathrm{p}<0.001$; PIPS: 105 seconds [82-141], p $<0.001$; PSEQ: 78 seconds [60101], p < 0.001; Table 5).

Assessing multivariable linear regression to control for relevant confounding variables with the QuickDASH, only the PROMIS-Pain Interference demonstrated a $\mathrm{p}$ value below 0.001 ( $\beta$ regression coefficient: 0.63 ; standard error: 0.10 ; partial $\mathrm{r}^{2}: 0.12$ ) (Table 6).

\section{Discussion}

Previous studies demonstrated that pain intensity and magnitude of disability are explained more by coping with pain rather than pathophysiology in patients with musculoskeletal disease [23, 24, 29]. Studies typically use a combination of pain-coping measures, which can be cumbersome and redundant. This study found evidence that the PROMIS-Pain Interference CAT, the PCS, PIPS, and PSEQ measure the same construct as indicated by a substantial correlation of all four questionnaires with an underlying-mathematically derived-trait. In other words, all of these measures are determined by an unobserved and unmeasured construct that has a strong influence on all of the measures. The PIPS questionnaire has the least floor

Table 4. Questionnaire score range, internal consistency, and floor and ceiling effect

\begin{tabular}{|c|c|c|c|c|c|c|c|c|}
\hline Questionnaire & $\begin{array}{l}\text { Number of } \\
\text { items }\end{array}$ & $\begin{array}{l}\text { Item completion rate } \\
(\%)\end{array}$ & $\begin{array}{l}\text { Median } \\
\text { (IQR) }\end{array}$ & Range* & $\begin{array}{l}\text { Possible } \\
\text { range }^{\dagger}\end{array}$ & $\begin{array}{l}\text { Cronbach's } \\
\text { alpha }^{*}\end{array}$ & $\begin{array}{l}\text { Flooring effect } \\
(\%)\end{array}$ & $\begin{array}{l}\text { Ceiling effect } \\
(\%)\end{array}$ \\
\hline PCS & 13 & 100 & $8.5(1-19)$ & $0-52$ & $0-52$ & 0.93 & $28(21)$ & $1(0.75)$ \\
\hline PIPS & 12 & 100 & $34(24-45)$ & $12-79$ & $12-84$ & 0.88 & $6(4.5)$ & $0(0)$ \\
\hline PROMIS-PI & 4-12 (CAT) & 100 & $56(51-62)$ & $39-75$ & $39-84$ & - & $16(12)$ & $0(0)$ \\
\hline PSEQ & 10 & 100 & $51(36-59)$ & $4-60$ & $0-60$ & 0.92 & $0(0)$ & $27(20)$ \\
\hline
\end{tabular}

* Range of scores patients per questionnaire; ${ }^{\dagger}$ possible range of scores per questionnaire; ${ }^{\dagger}$ represents the internal consistency; IQR = interquartile range; CAT = computerized adaptive testing; PCS = Pain Catastrophizing Scale; PIPS = Psychological Inflexibility in Pain Scale; PROMIS-PI = Patient-Reported Outcomes Measurement Information System-Pain Interference; PSEQ = Pain Self-Efficacy Questionnaire. 
Table 5. Time per questionnaire in seconds $(n=134)$

\begin{tabular}{llllllll}
\hline Questionnaire & Median (IQR) & Range* & $\mathrm{p}$ value & \multicolumn{2}{l}{ Multiple comparison ${ }^{\dagger}$} & & \\
\cline { 5 - 7 } & & & & & PCS & PIPS & PROMIS-PI \\
\hline PCS & $91(66-122)$ & $21-271$ & $<0.001$ & PCS & - & & \\
PIPS & $105(82-141)$ & $36-318$ & & PIPS & 0.0027 & - & \\
PROMIS-PI & $30(24-44)$ & $14-227$ & & PROMIS-PI & $<0.001$ & $<0.001$ & - \\
PSEQ & $78(60-101)$ & $24-316$ & & PSEQ & 0.017 & $<0.001$ & $<0.001$ \\
\hline
\end{tabular}

* Range of completion time patients per questionnaire; ${ }^{\dagger}$ Bonferroni correction of $\mathrm{p}$ value: $0.05 / 6=0.0083$; IQR $=$ interquartile range; $\mathrm{PCS}=\mathrm{Pain}$ Catastrophizing Scale; PIPS = Psychological Inflexibility in Pain Scale; PROMIS-PI = Patient-Reported Outcomes Measurement Information System-Pain Interference; PSEQ = Pain Self-Efficacy Questionnaire.

Table 6. Multivariable linear regression analysis with QuickDASH

\begin{tabular}{|c|c|c|c|c|c|c|c|}
\hline Questionnaire & $\begin{array}{l}\beta \text { regression coefficient } \\
(95 \% \text { confidence interval) }\end{array}$ & Standard error & $\mathrm{p}$ value & $\mathrm{R}^{2}$ & Variance Inflation Factor & Tolerance & Partial $\mathrm{R}^{2}$ \\
\hline PCS & -0.1297 & 0.0694 & 0.064 & 0.57 & 2.01 & 0.50 & 0.0117 \\
\hline PIPS & 0.0177 & 0.0606 & 0.771 & & 2.74 & 0.36 & 0.0003 \\
\hline PROMIS-PI & 0.6294 & 0.1039 & $<0.001$ & & 2.79 & 0.36 & 0.1227 \\
\hline PSEQ & -0.1912 & 0.0680 & 0.006 & & 3.07 & 0.33 & 0.0264 \\
\hline
\end{tabular}

QuickDASH = short version of the Disabilities of the Arm, Shoulder and Hand (DASH) questionnaire; PCS = Pain Catastrophizing Scale; PIPS = Psychological Inflexibility in Pain Scale; PROMIS-PI = Patient-Reported Outcomes Measurement Information System-Pain Interference; PSEQ = Pain Self-Efficacy Questionnaire.

and ceiling effects, but the PROMIS-Pain Interference questionnaire is the most efficient to complete.

There were several limitations. First, this study was performed in a tertiary care referral center for hand and upper extremity conditions. The subset of patients studied might therefore not be representative for the overall population with upper extremity conditions. On the other hand, the vast majority of our patients are direct referrals from primary care doctors. Second, questions within and between surveys seem very similar, which might have annoyed the subjects, resulting in less representative answers. We randomized the delivery order of the questionnaires to minimize this effect on our analysis. Third, for PROMIS-Pain Interference, we were not able to assess the internal consistency as a result of its CAT setup; therefore, we could not compare PROMIS-Pain Interference's consistency with those of the other three surveys. However, previous studies assessing the validity of the PROMIS-Pain Interference questionnaire demonstrated an internal consistency of $\geq 0.96$ (Cronbach alpha) [2], indicating substantial internal consistency.

We demonstrated a high factor loading of all four questionnaires, meaning that these questionnaires measure the same concept, as one would expect based on their purpose. Exploratory factor analysis is a statistical method used to uncover the underlying structure of a set of variables. This technique can be used to describe variability among observed, correlated variables in terms of a potentially lower number of unobserved variables called factors-to identify the underlying relationships between measured variables. In other words, relating to this study, are the four questionnaire scores all determined by a common underlying trait? This method of exploratory factor analysis has been used to individually validate PCS, PIPS, PROMIS-Pain Interference, and PSEQ [2, 14, 20, 30]. The four questionnaires are comparable in terms of the variables they measure individually; this is supported by the high collinearity among the questionnaires as demonstrated by the relative low partial $r^{2}$ of the individual explanatory variables as well as the multivariate multicollinearity statistics.

Interquestionnaire correlations were calculated to assess the relationship among the questionnaires. We found high correlation scores in our study. Previous studies were limited comparing only two of our four selected questionnaires: an interquestionnaire correlation of 0.62 was found between the PCS and a Spanish version of the PIPS [25] (in our study 0.70) and an interquestionnaire correlation of -0.72 was found between the PROMIS-Pain Interference and the PSEQ [17] (in our study -0.84).

In this study, internal consistencies of the PCS, PIPS, and PSEQ were high, whereas internal consistency for 
PROMIS-Pain Interference was not calculated as a result of the CAT administration method. Instrument coverage and reliability were good for all four questionnaires. Instrument coverage and reliability of a questionnaire-resulting in high internal consistencies-are precursors of instrument validity. Validity is the appropriateness of conclusions made on observations or measurements, specifically whether a test measures the intended trait [7]. Previous studies tested the validity of the PCS, PIPS, PROMIS-Pain Interference, and PSEQ [2, 19, 20, 22, 31]. Only one other study looked at instrument coverage in the PCS questionnaire and found a flooring effect of $32 \%$ and no ceiling effect [5] (versus $21 \%$ and $0.75 \%$, respectively, in our study).

The most effective way to obtain research data in the least amount of time is key in reducing both respondent and researcher burden, and we found that PROMIS-Pain Interference has an advantage. CAT provides a dynamic selection of items wherein the response to each item will guide the system's choice of the next item generating an individually tailored series of questions [2, 11, 12]. This results in lower questionnaire completion times because not every question needs to be answered. A previous study showed that it takes 45 seconds on average to complete the PROMIS-Pain Interference questionnaire [27] (in comparison to a median completion time of 30 seconds in our study).

Pain is one of the most common presenting symptoms in the practice of a hand and upper extremity surgeon [9, 21]. Hence, for a better measurement of a patient's health status, an understanding of the variation in pain for a given nociception and the influence of nociception on disability are key in clinical trials [23, 24, 29]. Finding the most effective way to measure coping strategies in response to nociception and magnitude of disability and avoiding redundancy is important. These measurements might help hand surgeons identify opportunities for psychological support in daily practice [26]. The widespread adoption of PROMIS-based questionnaires may not only lead to a reduction in respondent and researcher burden, but also in sample size requirements and ultimately study costs [17].

In conclusion, the various measures of coping strategies in response to nociception are all reducible to a single construct. We prefer the PROMIS-Pain Interference CAT over the PCS, PIPS, and PSEQ as a result of its simplicity, brevity, and relatively good coverage. The only caveat is that the PSEQ and PCS might be useful for a psychologist to use because in addition to the score, the individual questions can become talking points and foci of treatment.

Acknowledgments We thank the Harvard Catalyst for their statistical support.

\section{References}

1. Allegrante JP, Marks R. Self-efficacy in management of osteoarthritis. Rheum Dis Clin North Am. 2003;29:747-768, vivii.

2. Amtmann D, Cook KF, Jensen MP, Chen WH, Choi S, Revicki D, Cella D, Rothrock N, Keefe F, Callahan L, Lai JS. Development of a PROMIS item bank to measure pain interference. Pain. 2010;150:173-182.

3. AssessmentCenter. Available at: http://www.assessmentcenter. net. Accessed August 2014.

4. Beaton DE, Wright JG, Katz JN, Upper Extremity Collaborative Group. Development of the QuickDASH: comparison of three item-reduction approaches. J Bone Joint Surg Am. 2005;87:10381046.

5. Bot AG, Becker SJ, van Dijk CN, Ring D, Vranceanu AM. Abbreviated psychologic questionnaires are valid in patients with hand conditions. Clin Orthop Relat Res. 2013;471:4037-4044.

6. Bot AG, Nota SP, Ring D. The creation of an abbreviated version of the PSEQ: the PSEQ-2. Psychosomatics. 2014;55:381-385.

7. Cronbach LJ, Meehl PE. Construct validity in psychological tests. Psychol Bull. 1955;52:281-302.

8. Dworkin RH, Turk DC, Farrar JT, Haythornthwaite JA, Jensen MP, Katz NP, Kerns RD, Stucki G, Allen RR, Bellamy N, Carr DB, Chandler J, Cowan P, Dionne R, Galer BS, Hertz S, Jadad AR, Kramer LD, Manning DC, Martin S, McCormick CG, McDermott MP, McGrath P, Quessy S, Rappaport BA, Robbins W, Robinson JP, Rothman M, Royal MA, Simon L, Stauffer JW, Stein W, Tollett J, Wernicke J, Witter J, IMMPACT. Core outcome measures for chronic pain clinical trials: IMMPACT recommendations. Pain. 2005;113:9-19.

9. Hadler NM. Coping with arm pain in the workplace. Clin Orthop Relat Res. 1998;351:57-62.

10. Hudak PL, Amadio PC, Bombardier C. Development of an upper extremity outcome measure: the DASH (Disabilities of the Arm, Shoulder and Hand) [corrected]. The Upper Extremity Collaborative Group (UECG). Am J Ind Med. 1996;29:602-608.

11. Hung M, Nickisch F, Beals TC, Greene T, Clegg DO, Saltzman CL. New paradigm for patient-reported outcomes assessment in foot $\&$ ankle research: computerized adaptive testing. Foot Ankle Int. 2012;33:621-626.

12. Hung M, Stuart AR, Higgins TF, Saltzman CL, Kubiak EN. Computerized adaptive testing using the PROMIS physical function item bank reduces test burden with less ceiling effects compared to the short musculoskeletal function assessment in orthopaedic trauma patients. J Orthop Trauma. 2014;28:439-443.

13. Kennedy SA, Vranceanu AM, Nunez F, Ring D. Association between psychosocial factors and pain in patients with trigger finger. $J$ Hand Microsurg. 2010;2:18-23.

14. Lim HS, Chen PP, Wong TC, Gin T, Wong E, Chan IS, Chu J. Validation of the Chinese version of pain self-efficacy questionnaire. Anesth Analg. 2007;104:918-923.

15. Liu H, Cella D, Gershon R, Shen J, Morales LS, Riley W, Hays $\mathrm{RD}$. Representativeness of the patient-reported outcomes measurement information system Internet panel. J Clin Epidemiol. 2010;63:1169-1178.

16. Lozano-Calderon SA, Souer JS, Jupiter JB, Ring D. Psychological differences between patients that elect operative or nonoperative treatment for trapeziometacarpal joint arthrosis. Hand. 2008;3:271-275.

17. Menendez ME, Bot AG, Hageman MG, Neuhaus V, Mudgal CS, Ring D. Computerized adaptive testing of psychological factors: relation to upper-extremity disability. J Bone Joint Surg Am. 2013;95:e149. 
18. Morris LD, Grimmer-Somers KA, Louw QA, Sullivan MJ. Crosscultural adaptation and validation of the South African Pain Catastrophizing Scale (SA-PCS) among patients with fibromyalgia. Health Qual Life Outcomes. 2012;10:137.

19. Nicholas MK. The pain self-efficacy questionnaire: taking pain into account. Eur J Pain. 2007;11:153-163.

20. Osman A, Barrios FX, Kopper BA, Hauptmann W, Jones J, O'Neill E. Factor structure, reliability, and validity of the Pain Catastrophizing Scale. J Behav Med. 1997;20:589-605.

21. Pascarelli EF, Hsu YP. Understanding work-related upper extremity disorders: clinical findings in 485 computer users, musicians, and others. J Occup Rehabil. 2001;11:1-21.

22. Riley WT, Rothrock N, Bruce B, Christodolou C, Cook K, Hahn EA, Cella D. Patient-reported outcomes measurement information system (PROMIS) domain names and definitions revisions: further evaluation of content validity in IRT-derived item banks. Qual Life Res. 2010;19:1311-1321.

23. Ring D, Guss D, Malhotra L, Jupiter JB. Idiopathic arm pain. J Bone Joint Surg Am. 2004;86:1387-1391.

24. Ring D, Kadzielski J, Malhotra L, Lee SG, Jupiter JB. Psychological factors associated with idiopathic arm pain. $J$ Bone Joint Surg Am. 2005;87:374-380.

25. Rodero B, Pereira JP, Perez-Yus MC, Casanueva B, SerranoBlanco A, Rodrigues da Cunha Ribeiro MJ, Luciano JV, Garcia-Campayo J. Validation of a Spanish version of the psychological inflexibility in pain scale (PIPS) and an evaluation of its relation with acceptance of pain and mindfulness in sample of persons with fibromyalgia. Health Qual Life Outcomes. 2013;11:62.

26. Smith MV, Calfee RP, Baumgarten KM, Brophy RH, Wright RW. Upper extremity-specific measures of disability and outcomes in orthopaedic surgery. J Bone Joint Surg Am. 2012; 94:277-285.

27. Stapleton SJ, Degitz RJ. An innovative data collection method for investigating unresolved pain after ED discharge: a pilot study. J Emerg Nurs. 2014;40:598-604.

28. Sullivan MJL, Bishop SR. The pain catastrophizing scale: development and validation. Psychol Assess. 1995;7:524-532.

29. Vranceanu AM, Barsky A, Ring D. Psychosocial aspects of disabling musculoskeletal pain. J Bone Joint Surg Am. 2009;91: 2014-2018.

30. Wicksell RK, Lekander M, Sorjonen K, Olsson GL. The Psychological Inflexibility in Pain Scale (PIPS)-statistical properties and model fit of an instrument to assess change processes in pain related disability. Eur J Pain. 2010;14:771 e771-714.

31. Wicksell RK, Renofalt J, Olsson GL, Bond FW, Melin L. Avoidance and cognitive fusion-central components in pain related disability? Development and preliminary validation of the Psychological Inflexibility in Pain Scale (PIPS). Eur J Pain. 2008;12:491-500. 